\title{
Akkaraman koyununda articulatio temporomandibularis ve çiğneme kaslarının anatomisi*
}

\author{
Murat KABAK \\ Mustaf
}

\begin{abstract}
Özet: Akkaraman koyununda articulatio temporomandibularis ve çiłgneme unsurlarının morfolojik yapısmm belirlenmesi amacıylat yapılan çalışmada, 8 ade ergin Akkaraman koyun başı incelendi. Adı geçen hayvanların 4 adet bașs. cklem ve disscus. articularis in histolojik olarak incelenmesinde, diğer başlar ise çigneme kasları ve cklemin makroskobik bulguları için kullanıldı. Eklemin kemiksel yapıssın oluşturan caput mandibulac ve fossa mandibularis'in latero-medial yönde oldığu belirlendi. Articulatio tcmporomandibularis'i iki bağımsiz boşluğa ayıran discus articularis’in fibro-kartilaginöz. yapıda olduğu ve cklem kapsuliune

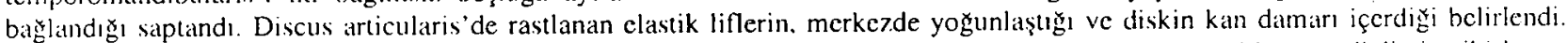
Ciọncme kaslarından $m$. masscter iiç, $m$. temporalis bir, $m$. pterygoidcus lateralis ve musculus pterygoideus medialis isc iki kısum olarak incelendi. Sonuç olarak. Akkaraman koyununda articulatio temporomandibularis ve çiğneme kaslarımn morfolojik dectaylarn ortaya konmuştur

Anahtar kclimeler: Articulatio temporomandibularis, çiðneme kasları, discus articularis, mandibulat
\end{abstract}

\section{The anatomy of the articulatio temporomandibularis and masticatory muscles of the Akkaraman sheep}

Summary: The aim of the study was to establish the morphological structure of temporomandibular joint and the masticatory clements in the Akkaraman shecp. In the study, 8 native Akkaraman shecp were used. The heads of four sheep were used to examine the joint and discus articularis histologically. The other heads were used for macroscopic examination of the masticatory muscles and the joints. It was detcrmined that the orientations of the caput mandibulac and fossa mandibularis, which form the bone stucture of the joint were in latero-medial direction. It was observed that the joint divided in two compartments by discus articularis. which wals connected to the catpsula articularis. Discus articularis was fibro-cartilaginous structure and elastic fibres were seen densely and located preferentially in the center of the articular disc. The discus articularis included blood vessels. The masticalory muscles were found to include four muscles. Musculus masseter were divided into three parts. whereas in. temporalis wats single part. Both $\mathrm{m}$. pterygoidcus lateralis and $\mathrm{m}$. pterygoideus medialis were divided into two parts. Consequently. morphologic characterizations of temporomandibular joint and masticatory muscles in the Akkaraman sheep were established by the present study

Key words: Articular disc, mandible, masticatory muscles, temporomandibular joint

\section{Giriş}

Akkaraman koyunu Artiodactyla takımının Bovidae familyasma dahil olup besinlerinin tamamı bitkiseldir. Momelilerin farkl beslenmelerinden dolayı, articulatio lemporomandibularis'i şckillendiren unsurlarda, çiğneme kaslarında ve buna bağlı olarak mandibular harekctlerde de farklılıklar görülebilir $(3,10,15)$.

Inkongruent ginglymus olarak çalışan articulatio temporomandibularis'in cklem yüzleri arasındaki uyununu fibrö ya da fibro-kartilaginöz yapıda olan discus articularis sąglar $(11,12,14,19,21)$. Adı geçen oluşum makroskobik $(11,14,20,21)$ ve mikroskobik $(12,14,15,23)$ olarak incelenmiştir. Evcil memelilerde ve insanda articulatio temporomandibularis, cklem yüzlcrinin şekline göre condylo-meniscal ve menisco-temporal olarak alt iki ekleme ayrılır. Eklem; insan, domuz, köpek, sıçan ve tavşanda condylo-meniscal, koyun, keçi ve sığırda menisco- temporal olarak şekillenir (3). Bazı araşturıcılar eklem yüzlerinin uyumuna ve yapisina bakarak insan $1 \mathrm{~cm}-$ poromandibular eklemine en yakın olan hayvanın domuz. olduğunu $(3,25)$, diğger araştırıcılar ise buiyuikliik, şckil ve yapı bakımından koyun ve keçinin insana daha çok benzediğini ve dencysel calışmalarda bu hayvanlarm kullanılabileceğini belirtmişlerdir (4-6,16). Articulatio temporomandibularis'de açılıp-kapanma (rotasyon). ileri-ģeri (kayma) ve yanal (lateral) hareketler şeklinde uiç farklı çiğneme hareketi görülür $(1,19)$. Lateral hareket özellikle insan ve ruminantia' da gelişmiştir (11,12.21).

Evcil memeli hayvanlarda çiğneme kasları m. masseter, $m$. temporalis, $m$. pterygoideus medialis ve $m$. pterygoideus lateralis olarak incelenir $(17,21,24,26)$.

Bu çalışma, Akkaraman koyununda articulatio temporomandibularis ve çiğneme kaslarınn morfolojisini de taylı olarak incelemek anıacıyla yapılmışır.

Bu çalısma Ankara Üniversitesi Araşurma Fonu tarafından 96.30.00.24 proje numarası ile desteklenen doktora tezinden bir bölüin özetidir. 


\section{Materyal ve Metot}

Çalışmada, 8 adet ergin Akkaraman koyun başı kullanıldı. Hayvanlarda kondrodistrofi, hastalık ya da çcnenin açılıp kapanmasında bir sorun görülmedi. Histolojik incelemelerde kullanlacak başlarm (4 adet) perfuzyon işlemleri yapıldıktan sonra, diğer başlarla birlikle \% 10'luk formol havuzuna konarak fikzasyonları sağlandı.

Tüm başlar median hattan kesilerek ikiye ayrıldı. Daha sonra articulatio temporomandibularis ve çiğneme kasian makroskobik olarak incelendi. Çiğneme kaslarının lil yönleri kafa dizzlemine göre isimlendirildi. Lif seyirleri. discus articularis ve eklem stereomikroskop (Olympus MTX marka) ile incelendi. Histolojik incelemeler için discus articularis”ler lateral, central ve medial olmak üzere iiç kısma ayrıldı. Eklemin oluşununa katılan kemik dokular ise formik asit-Na sitrat (18) metodu ile dekalsifiye edildi. Discus articularis lerden horizantal, eklemclen ise sagittal olarak alınan 5-6 mikron kalınlığındaki ömeklerden histolojik preparatlar hazırlandı. Discus articularis'de bulunan kollagen demetler Weigert's elastik boyama yöntemi (18), elastik iplikler Orcein boyama yöntemi (9) ile belirlendi. Eklem ise Crossmon (8) un ïçlï boyama yöntcmi ilc buyandı. Tün preparatlar 1 şk mikroskobunda incelendi.

Anatomik terimlerde Nomina Anatomica Veterinaria (22) esas alınd.

\section{Bulgular}

\section{Articulatio temporomandibularis}

Occlusial (çiğneme) düzlemin yukansında yer alan temporomandibular eklemin, caput mandibulae ve fossa mandibularis'deki cklem yuizlerinin latero-medial yönde

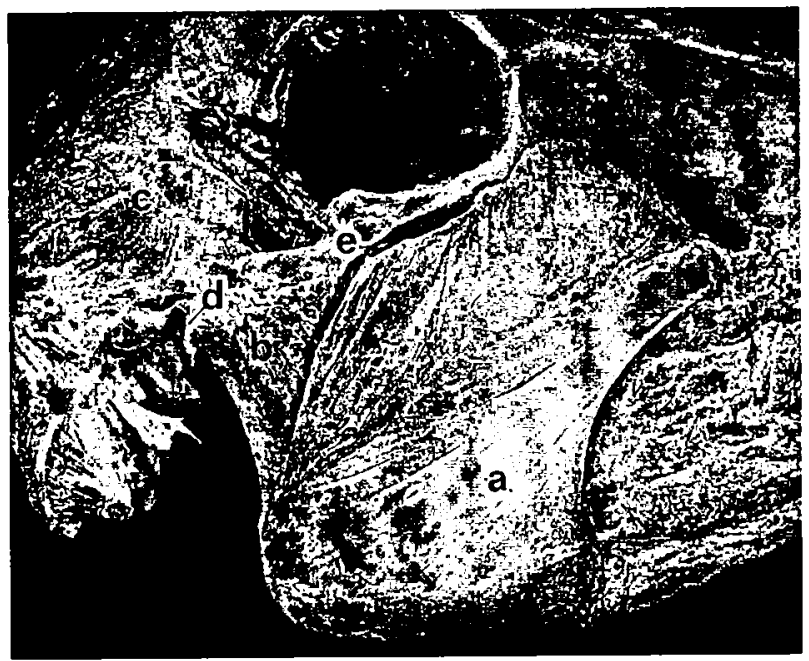

Şckil I. Giğneme kaslarnmu lateral den görïniumü. Figure 1. L atteral view of the maticitory muscles.

a- m. masseter. pars superficialis. b- m. massecer, pars profunda, c- Im. lemporalis, d-capsulat articularis, c-arcus zygomaticus.

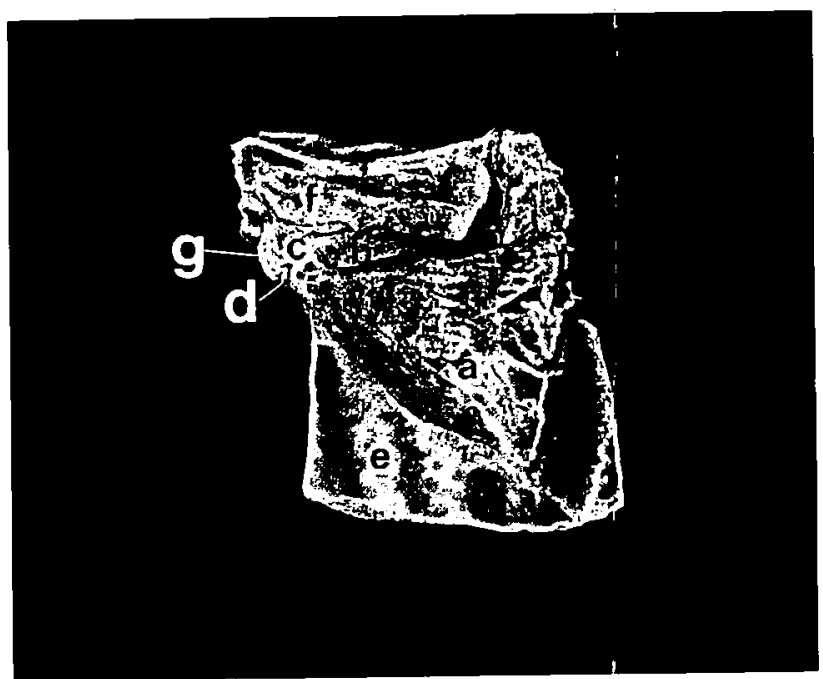

Șckil 2. Çiğneme kaslarının medial 'den göriiniumi

Figure 2. Medial vicw of the masticattory muscles. a- m. pterygoideus lateralis'in ventral parçass (the ventrall part of lateral pterygoid muscle), b- m. pterygoideus latcralis in dorsal parçass (the dorsal part of lateral pterygoid muscle). c-capsula articularis, d- discus articularis, c- ramus mandibulac. f- os temporale. g- cklemin caudal'i (caudal part of jointi.

ve düzc yakın olduğu saptand. Articulatio tenıporomandibularis'in ince bir eklem kapsulası (Şekil Id. $2 \mathrm{c}$ ve 4k) ile örtülü olduğu, caudal’inin yağ ve bağg dokusu. diğer bölümlerinin ise çiğneme kasları ile desteklendiğ belirlendi.

Discus articularis: Discus articularis' in (Şekil 2d ve 4d) cklem kapsulasına bağlanarak cavum arlicularc' yi iki bağımsız boşluğa ayırdığı göruildui (Şekil 4). Eklem yüzlerine uyan discus articularis in merkezinin ince. kenarlarının kalın olduğ saptand. Discus articularis dekj en kalın bölümün rostral uç olduğu gözrlendi (Şckil 4a). Adı geçen oluşumun kollagen demetler (Şekil 6b) ve klkırdak hücrelerinden (Şckil 6a) meydana geldiğj, ayruca merkezi kısımda yoğun, rostral ve caudal uçla a\% miktarda clastik ipliklerin (Şekil 5c) de hulunduğu belirlendi. Discus articularis'in hemen her yerinde kan damarlarma rastland 1

\section{Çiğneme kasları (Mm. masticatores)}

Çiğneme kasları m. masscter, m. temporalis. $\mathrm{m}$. pterygoideus medialis ve $\mathrm{m}$. pterygoideus lateralis oliarak incelendi.

Musculus masseter: Ų̧ kısmdan moydana gelen bu kasın pars superficialis'i (Scekil la) tuber faciale, crista lacialis ve os zygomaticum un lateral'inden bassladig ve ramus mandibulae'nin ventral kenarı ile angulus mandibulae'de sonlandığı belirlendi. Lif yönuinuiun caudoventral olduğu göruildü. Pars internedia'nun. crista fatcialis'in ventral'inden ve os zygomaticum un ventrolateral yüzünden başladığı ve ramus mandibulace nin la. teral yüzüne bağlandı ğ belirlendi. Kas liflerinin ventral ${ }^{\circ} \mathrm{c}$ 


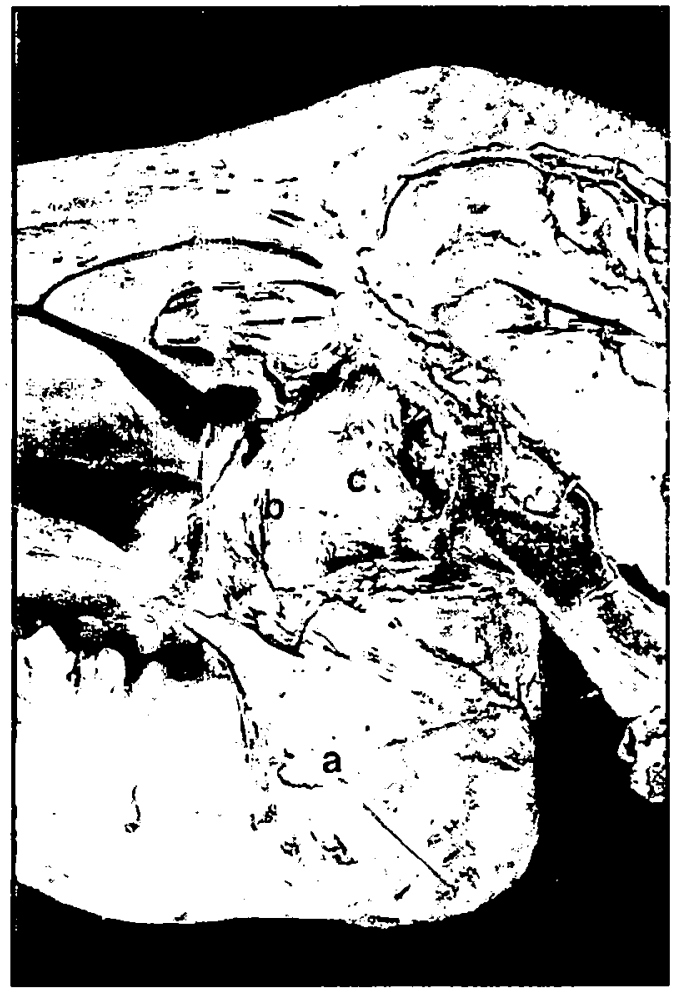

Sckıl 3. Ciŏncme kaslatrum medial den görünumii. Figure 3. Medial view of the masticatory muscles. a- $m$. plerygoideus medialis' in medial parçası (the medial part of medial pterygoid muscle), b- m. pterygoideus medialis'in lateral parçası (the lateral part of medial pterygoid muscle), c.. m. pterygoideus lateralis.

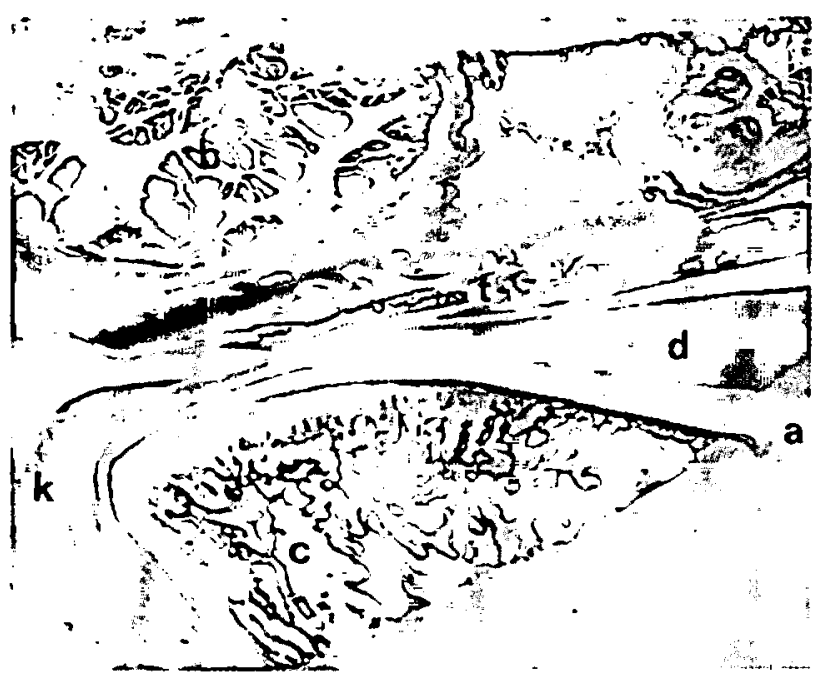

Şekil 4. Articulatio temporomandibularis. Crossmon'un üçlï boyamasi, $\times 12$

Figure 4. Temporomandibular joint. Crossmon's triple stain, $x 12$. a- anterior, b- m. temporalis, c-caput mandibulae, d- discus arlicularis, k- capsula articularis, t- os temporalc.

doĝ̣ru seyrettiği tespit edildi. Pars profunda'nın (Şekil lb), arcus zygomaticus'un ve os zygomaticum'un ventral kenaryyla, medial yüzünden başladığı vi nervus massetericus ile biri rostral, dið̌eri caudal iki parçaya ayrıldı̆̆ı görüldü. L.ifleri vertikal olarak seyreden rostral'deki par-

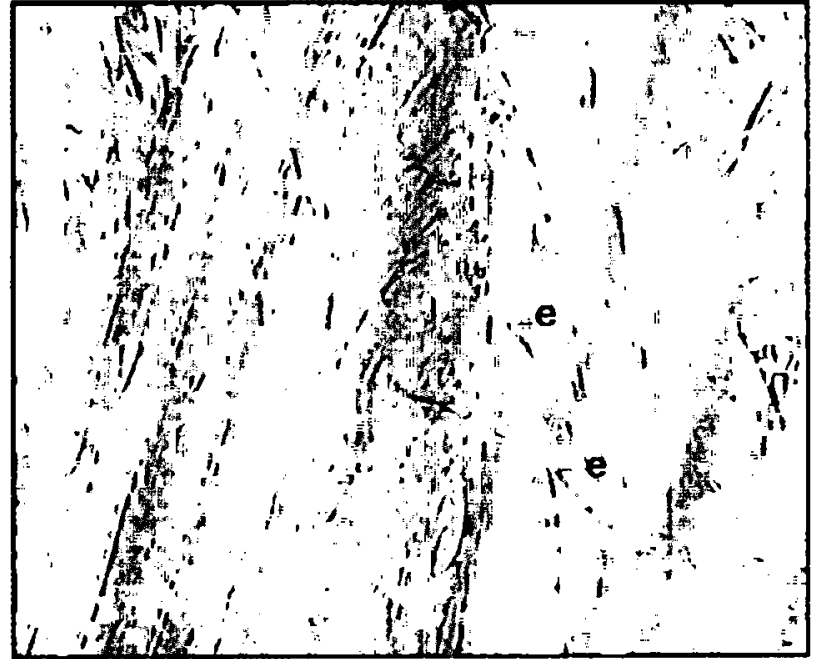

Şekil 5. Discus articularis'de clastik ipliklerin görünümij. Orcein boyama, $\times 320$.

Figure 5 Appearance of elastic fibers in articular disc. Orcein stain, $\times 320$ ). e- clastik iplikler (clastic fibers).

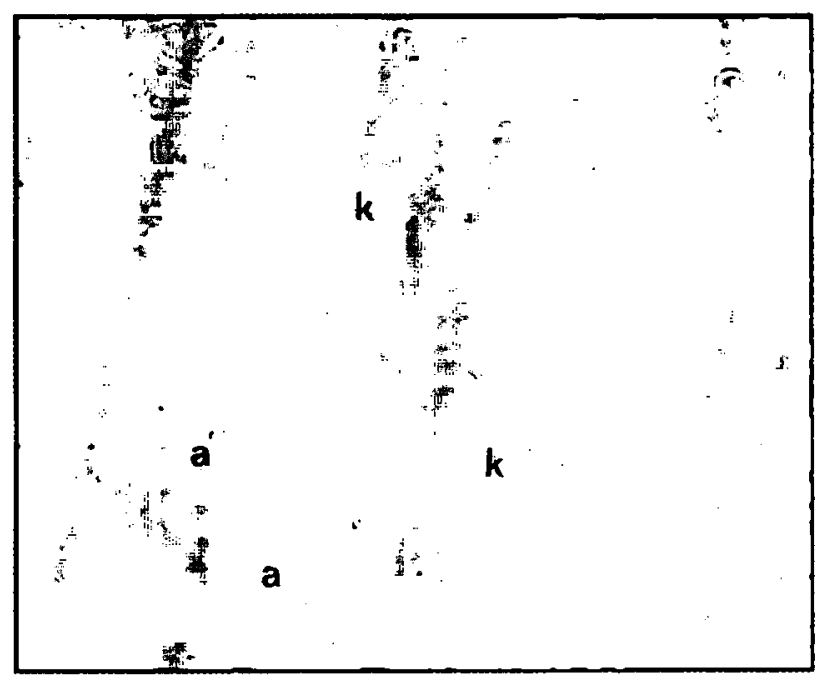

Şekil 6. Discus articularis'de fibro-kartilaginöz doku. Weigert'in clastik boyaması. $\times 29()$.

Figure 6. The fibro-cartilage tissuc in articular disc. Weigert's elastic stain, x290. a- kıkırdak hücresi (cartilage ccll).

k- kollagen demetler (collagen bunches).

çanın bazı lifleri m. temporalis ile birlikte processus coronoidcus'un rostral ucuna ve lateral yüzzune. lif yönnii rostro-ventral olan caudal'deki parçanın ise fossa masseterica' ya bağlandı ̆̆ belirlendi.

Musculus temporalis: Bütün bir kas olarak incelenen m. temporalis'in (Şekil 1c) zayıf olarak şekillendiği gözlendi. Fossa temporalis'den başlangı̨̧ alan kasın bazı liflerinin processus coronoideus'un latcral'inde, diğerlerinin ise aynı oluşumun medial'inde scyrcttiği tespit edildi. Lalteral'de seyreden liflerin ramus mandibulae'nin rostral ve lateral kenarına, medial'deki liflerin ise ramus mandibulae'nin medial yüzüne ve foramen mandibulac'nin ventral'ine bağlandığı belirlendi. 
Musculus pterygoideus lateralis: Iki bölüm halinde incelenen m. pterygoideus lateralis' in (Şckil 3c) kalın ve ventiral'de bulunan parçasının (Şekil 2a), os pterygoideum ile os basisphenoidale'nin ala'snum lateral yüzünden başlangı̧̧ aldığı belirlendi. Bu kısmın liflerinin, condylus mandibulae'nin rostral ucunda ve fovea pterygoidea'da sonlandığı gözlendi. Kasın dorsal'de ve ince olan parçasının (Şekil 2b), os basisphenoidale'nin ala'sı ile os temporale'nin pars squamosa'smn margo sphenoidale si sımıından başladığı ve kavisli bir şekilde seyrederek, condylus mandibulac' nin medial ucunda eklem kalpsulasina hağlandığı tespit edildi (Şekil $2 \mathrm{c}$ ). Her iki parça liflerinin sagittal seyirli olduğu saptandı.

Musculus pterygoideus medialis: Musculus pterygoideus medialis'in, medial ve lateral olarak iki kısma aynldı num'un lanina horizantalis' inin margo liber'i ile aynı kemiğin lamina perpendicularis'inin tabanmdan, ayrica os pterygoidcum'un hamulus pterygoidcus'u ve os basisphenoidale'nin processus pterygoideus'unun ventral kenarindan başladiğı görülldii. Lif yönleri caudo-ventral olan bu kısmin, ramus mandibulae'nin ventral kenarı ile angulus mandibulae'nin medial yüzüne iki ayn bölüm olarak bağländı̊̆ tespit edildi. Lateral kısmın (Şekil 3b) jse os palatinun ín lamina perpendicularis'i ile os pterygoideum'uin lateral yúzüunden başladığ ve ramus mandibulae ile corpus mandibulac sımırına bağlandığı gözlendi.

\section{Tartışma ve Sonuç}

Besinlerini bitkisel kaynaklardan sağlayan Akkaraman koyununda articulatio temporomandibularis ve çiğneme kaslarının anatomisi incelenmiştir.

Articulatio temporomandibularis literatürde $(14,15)$ ifade edildiği gibi, occlusial (çiğneme) düzlemin yukarısınça göruilmuişstur. Ince bir cklem kapsulası ile çevrili olan articulatio tenporomandibularis'in caudal'inin, gevşek bağ dokusu ile (4), diğer kısımlarının ise çiğneme kasları tarafından desteklendiği belirtilmiştir $(2,12,21,24$, 28). Yapılan çalışmada da benzer bulgular elde edilmiştir.

Articulatio temporomandibularis in eklem yuizleri arasında yer alan discus articularis'in bazı araşturicılar (14.20) tarafinda da ifade edildið̧i gibi merkezinin ince kenarlarının kalın şekillendiği görülmüşüur. Gillbe (14) 'nin disktcki kalınlığın caudal'de fazla olduğunu bildirmesi bu çalışmaya uymamaktadır. Aynı araşıırıcı (14) discus articularis' in lateral'de caput mandibulae'ye, medial'de os temporale'ye, bazı araşuncılar ise $(12,21)$ diskin eklem kapsulasına bağlandığın bildirmişlerdir. Yapılan çalışmada discus articularis'in eklem kapsulüne bağlandığı görülmiiş̧tür. Discus articularis'deki fibro- kartilaginöz yapı, kollagen demetlerin seyri ve elastik ipliklerin dağ $\mathrm{g}_{1} \mathrm{l}_{1}$ I literatüre $(14,15,23)$ benzerdir. Incelenen hayvanlarda discus articularis' in hemen her yerinde kan damarları görülnüustür. Gillbe $(14,15)$ ile Bosanquet ve Goss (5) kan damarlarının sadece diskin rostral' inde vas. culer sinuzoid tarzındia bulunduğunu ifade etmişslerdir. Bifano ve ark. (4) keçide diskin caudal bağlantısında da kan damarlarının varlığını bildirmiştir. Yapılan çalışmada vassculer sinuzoid tarz.ında damarlaşma görïlememiştir.

Çiğneme kasları literatürde $(17,24,26)$ tanımlandığ gibi m. masseter, m. temporalis. m. pterygoidcus lateralis ve m. pterygoideus medialis olarak incelenmişcir

Musculus masseter'in pars superficialis. pars intermedia ve pars profunda olarak uiç parçaya ayrıldığı ve bu üç bölümün başlangıçı, sonlanması ve lif yönlerinin literatüre (13) benzer olduğ belirlenmiştir. Musculus temporalis literatüre (13) uygun bir şckilde büttuin bir kas olarak incelenmiştir. Ibrahinı ve ark. (17) adı geçen kası m. temporalis superficialis ve $\mathrm{m}$. temporalis profundus olarak ikiye ayırmışlar ve m. lemporalis superlicialis" in pro. cessus coronoideus'un lateral'inde, m. temporalis pro. fundus'un ise processus coronoidcus' un medial' 'inde seyrettiğini bildirmişlerdir. Getty (13)'nin koyunda biituin bir kas olarak belirttiği m. pterygoideus mediâlis yapılan çalışmada, Ibrahim ve ark. (17)'nın bildirdiklerine belı/er şekilde iki yaprak olarak incelenmiştir. Musculus pterygoideus lateralis Getty (13) ile Ibrahim ve ark. (17)'dan farklı olarak iki böliinde incelenmiştir. Kalın ve ventral'de olan parçanın, Getty (13)'nin bildirdiğ j̧ckilde, incisura mandibulae'nin medial'inde kalan condylus mandibulae'nin rostral'ine ve fovea pterygoidea'ya, ince ve dorsal'de olan parçanın ise articulatio temporomandibularis'in medial ucunun eklem kapsulasina bağlandığ görülmüş̧ür. Musculus pterygoideus lateralis" in bu yalpısı, insanda ayn kası tanmmlayan araşuncıların (7,24) ifadclerine benzer olmasma rağmen, ince ve iistte seyreden kas parçasının discus articularis'e bağlanmaması farkhlığı oluşturmuştur.

Sonuç olarak, Akkaraman koyuntunda articulatio) temporomandibularis'in oluşumuna katılan caput mandibulae ve fossa mandibularis in düze yakın olması. özellikle lateral çiğneme hareketinin yapılabildiğini gösterir. Fibro-kartilaginöz yapıda olan discus articularis'de. elastik liflerin oldukça fazla olması eklem üzerine diş̧en kuv vetlere karşı diskin dayanıklılığını arturır. Eklemin caudal kenarı yağ ve bağ dokusu ile desteklenmiş̧ir. Bu nedenle, eklem içi uygulamalar daha kolay gerçekleş̧irilir. Musculus masseter uiç, m. temporalis bir. m. pterygoideus medialis ve m. ptcrygoideus lateralis iki bölim olarak görülmüştür. Özzellikle m. pterygoideus lateralis"in iyi gelişmiş olması, lateral harekctlerin daha guiçlii yatpıldığın gösternektedir. 


\section{Kaynaklar}

I. Aruncı K, Elhan A (1995): Anatomi. Kemikler, Eklemler, Kaslar ve ļ: organlar: 1. Cilt, Güneş Kitap Evi Lid Şti, Ankara.

2. Bade H, Schenck C, Koebke J (1994): The function of discomuscular relationships in the human temporromandihular joint. Acta Anat, 151. 258-267.

3. Bermejo A, Gonzalez O, Gonzalez JM (1993): The pig as an animal inodel for experimentation on the temporomanditular articular complex. Oral Surg Oral Med Oral Pathol. 75, 18-23.

4. Bifano C, Hubbard G, Ehler W (1994): A comparison of the form and function of the human. monkey and goat temporrmandibular joint. J Oral Maxillofac Surg, 52. 272275.

5. Bosanquet AG, Goss AN (1987): The sheep as a model for temporomandibular joint surgery. Int J Oral Maxillofac Surg. 16, 600)-603.

6. Bosanquet AG, Ishimaru JI, Goss AN (1991): Effect of facia repair of the temporomandibular joint disc of sheep. Oral Surg Oral Med Oral Pathol. 72, 520-523.

7. Carpentier P, Yung JP, Bonnet RM, Meunissier M (1988): Insertions of the lateral pterygoid muscle: An anatomic study of the human temporomandibular joint. J Oral Maxillofac Surgy, 46, 477-482.

8. Crossmon (G (1937): A modification of Mallory's connective tissue stain with a discussion of the principles invelied. Anat Rec. 69. 33-38.

9. Culling CFA, Allison RT, Barr WT (1985): Cellular Pathology Technique. Fourth Ed. Butterworths, London.

10. Demirsoy A (1992): Yaşamin Temel Kurallart. Omursaltar (Sürüngenler. Kuslar ve Memeliler). Cilt 3, K1sim 2. Bölüm 42. Birinci Baskı. Mcteksan A\$, Ankara.

1 I. Dyce KM, Sack WO, Wensing C.JG (1987): Teexhook of Veterinary Anatomy: WB Saunders Company, Philadelphia.

12. Fenoll AB, Sequeros OG, Gonzalez JMG (1992): Hisrological study of the temporomandibular joint capsule: Theory of the articular complex. Acta Anat, 145, 24-28.

13. Getty R (1975): The Anatomy of the Domestic Animals. Fifth Edition. $V_{0} 1$ and 2. WB Saunders Company, Philadelphia.

14. Gillbe GV (1973): A comparison of the disc in the craniomanditular joint of three mammals. Acta Anat. 86, 394-41)9.
15. Gillbe GV (1975): The funtion of the dise of the temporomandibular joimt. J Prosthet Dent. 33. 196.204

16. Goss AN, Bosanquet AG (1989): An amimal model for tmi arthroscopy. J Oral Maxillofac Surg. 47. 537-538.

17. Ibrahim IA, Yousif MJ, Hassan NH (1992): Comparative anatomical study on the muscles of mastication of gorts and sheep in Mosul, Iraq. Iraqi J Ver Sci. 5. 31-41. 'Alinmışur' Vet Bull. 1993. Abstr: 5496.

18. Luna LG (1968): Manual of Histologic Stanimg Methods of the Armed Forces Institute of Pathology: Third Edition. McGraw-Hill Book Company, New York.

19. McKay GS, Yemm R, Cadden SW (1992): The structure and function of the temporomandihular joint. Br Dcul $\mathrm{J}$. 173, 127-132.

20. Meister VR, Berg R (1974): Untersuchungen uber die form. GrïBe und dicke der disci articulares articulationis temporomandibularis von rind. schal. schwein and hund. Anat Anz, 135. 24-34.

21. Nickel R, Schummer A, Seiferle H (1986): The Anatrm of the Domestic Animals. Vol 1. Verliag Palul Parcy. Berlin

22. Nomina Anatomica Veterinaria (1994): Puhlished by the International Committees on Veterinan Gross Anatomical Nomenclature. Fourth Ed. New York.

23. Ortuğ G (1988): Çene ekleminin discus articularis'inde rasterelektronen mikroskopik (Scamning) arasstumalar. Anadolu Üniv Tıp Fak Yayınları. No 3. Eskişchir.

24. Schmolke C (1994): The relationship between the tem. poromandibular joint capsule. aricular disc and jaw mass. les. J Anat, 184. 335-345.

25. Ström D, Holm S, Clemensson S, Haraldson T (1986): Gross anatomy of the mandibular joint and masticatory. muscles in the domestic pig (Sus scroffa). Arch Oral Biol. 31. 763-768.

26. Velasco JRM, Vazquez JFR, Collado J.J (1993): The relationships hetween the temporonnandibular join dise and related masticatory muscles in humans. J Oral Maxillolitc Surg. 51, 39()-395.

Geliş tarihi : 17.9.2001/Kabul tarihi : 10.10.2001

Yazışma adresi:

Yrd.Doģ:Dr.Murat Kahak

Mustafa Kemal Universitesi. Veteriner Fakiallesi

Anatomi Anabilion Dall, Antakva, Hatav.

e-mail: muratkabak@ mku.edu.tr 